\title{
Signal Detection Based on Walsh Transform for Spectrum Sensing
}

\author{
Guoxiang Yang, Guangliang Ren, Kun Wu \\ State Key Lab. Of Integrated Services Networks, Xidian University, Xi’an China \\ Email: gxyang07@126.com \\ Received July, 2013
}

\begin{abstract}
Spectrum sensing is a key technology to improve spectrum efficiency. In this paper, we propose a novel signal detection method based on Walsh transform for spectrum sensing. The main idea behind is that the received signal is transformed into another domain by Walsh transform and the test statistic is obtained by exploiting the feature of the useful signal in the new domain. The new method can perform well at low signal-to-noise rate (SNR). Simulation results show that the proposed method has better performance than the spectral feature detection based on power spectrum.
\end{abstract}

Keywords: Cognitive Radio; Walsh Transformation; Feature Detector; Low SNR

\section{Introduction}

Due to the rapid development of wireless communication, spectrum scarcity has become an urgent problem with the increasing proliferation of wireless devices and services. Cognitive radio (CR) is an emergent technology to solve this problem, which can allow the unlicensed users to opportunistically access the spectrum assigned to the licensed users without harmful interference to the current users. Spectrum sensing is one of the most important functionalities of $\mathrm{CR}$, which can reliably detect the existence of the user on the current bands.

In general, there have been several popular signal detection methods for spectrum sensing such as energy detection[3], coherent detection (matching filter) and feature detection[4,5]. Energy detection is commonly used, as it doesn't require prior knowledge about the correlation structure of the primary signal. However, the energy detector requires perfect information for the noise variance to properly perform the detection. If a pre-set pattern of primary signals is known to the receiver, the coherent detector is usually applied. The above two approaches are simple and effective, but they cannot detect weak signals at very low signal-to-noise rate (SNR). Feature detector is usually used when some information of the transmitted signal is priori known. The spectral feature detector based on power spectrum of the signal is

\footnotetext{
${ }^{*}$ This work was supported in part by the State Natural Science Foundation of China, Grant No.61072102 and National Major Specialized Project of Science and Technology, Grant No.2011ZX03001-007-01. The authors are with the State Key Laboratory of Integrated Service Networks, Xidian University, Xi’an 710071, China.
}

one example of the feature detectors, which exploits the unique spectral pattern of a specific signal to detect its presence. The spectral feature detector outperforms the energy detector and the coherent detector.

In this paper, we study a feature detector based on Walsh transform. Firstly, the received signal is processed by Walsh transform, that is, the signal is transformed into another domain. Then, the test statistic is obtained by exploiting the feature of the useful signal in the new domain, which is compared with the threshold to decide the presence of the useful signal. The new method is applicable to signals with rectangle envelope and robust to white noise. The main contribution of this paper is that we introduce the principle of how to improve the performance of signal detection and propose a novel practical method which has much better performance than the spectral feature detector.

The paper is organized as follows. In section 2 the system model is introduced. In section 3 our method is described, and the performance is analyzed. In section 4 the new detector is simulated and compared with the spectral feature detector. Section 5 concludes the paper.

\section{System Model}

As the goal of detecting primary user (PU) signals is to ascertain their presence or absence in the licensed frequency band, it is usually formulated as a binary hypothesis testing problem. It is assumed that the modulation type and the symbol rate are known,

$$
\begin{array}{ll}
H_{0}: \quad r(t)=n(t) \\
H_{1}: \quad r(t)=x(t)+n(t)
\end{array}
$$


where $H_{0}$, represents the absence of the PU signal $x(t)$ and the received signal $r(t)$ contains only additive white Gaussian noise (AWGN) with zero mean and variance $\sigma^{2} . H_{1}$ stands for the presence of the PU signal in the band, and $r(t)$ consists of the primary signal $x(t)$ corrupted by noise $n(t)$. The baseband signal $x(t)$ is formulated as

$$
x(t)=a(t)=\sum_{n=-\infty}^{\infty} a_{n} g_{T}\left(t-n T_{b}\right),
$$

where $a_{n}$ is the symbol sequence, $g_{T}(t)$ is the rectangle envelope of symbols, $T_{b}$ is the symbol period.

\section{Proposed Algorithm}

Feature detectors usually have good performance for the reason that they exploit the typical feature of the PU signal which is different from that of noise. For example, the spectral feature detector makes use of the feature of the signal in frequency domain. In order to improve the detection performance, the received signal needs to be transformed into other domain, in which the feature of the PU signal is more obvious. When the transformation is orthogonal, if the obtained nonzero coefficients of the PU signal after transformation are less, i.e., the PU signal can be represented by less components of orthogonal functions, the feature of the PU signal in the new domain is more obvious. Then for the detection of signals whose baseband signals have rectangle envelope, we introduce the Walsh transform to process the signal, which is also an orthogonal transformation.

It is assumed the duration time of the received signal $r(t)$ is $T$, but in order to making the derivation simple, we regard $T$ as 1 . The signal $r(t)$ which is integrable is capable of being represented by a Walsh series defined over the open interval $(0,1)$ as

$$
r(t)=c_{0}+c_{1} W A L(1, t)+c_{2} W A L(2, t)+\ldots,
$$

where the coefficients are given by

$$
c_{k}=\int_{0}^{1} r(t) W A L(k, t) d t,
$$

and the Walsh series $W A L(k, t)$ is derived from

$$
\begin{aligned}
N & =2^{p}, W A L(k, t)=\prod_{r=0}^{p-1}(-1)^{k_{p-1-r}\left(t_{r}+t_{r+1}\right)} k \\
& =1,2, \ldots, N,
\end{aligned}
$$

where $t$ and $k$ are the arguments of the function expressed in binary notation.

The obtained coefficients $\left\{c_{k}\right\}$ form a vector $C$,

$$
C=\left[c_{0}, c_{1}, c_{2}, \ldots\right]
$$

which can represent the signal $r(t)$ in the new domain. Figure 1 shows an example of BPSK baseband signals with rectangle envelope and Figure 2 shows the obtained vector of the BPSK baseband signal after Walsh transformation. From the figures it is illustrated that the BPSK signal can be represented be limited components of the Walsh functions.

Then, assuming that the vector of the signal $x(t)$ is $C_{\text {ideal }}$ which is normalized, the following correlation formula can be applied to detect the presence of $x(t)$ :

$$
S=C_{\text {ideal }} C^{T} \underset{H_{0}}{\stackrel{H_{1}}{<}} t,
$$

where $S$ is the teat statistic, $(\bullet)^{T}$ is transposition operation and $t$ is the decision threshold. If $S$ is greater than the threshold, $H_{1}$ is decided, i.e., presence of the signal $x(t)$; otherwise, $H_{0}$ is decided, i.e., absence of the primary signal.

In practical application, only discrete signal can be dealt by computer, so we consider the performance of the detector based on discrete Walsh transformation. Assuming that the total sample points of the receiver signal $r(t)$ is $N$ and that the oversample ratio is $m$, the obtained points after discrete Walsh transformation is also $N$. When there is no noise in the signal, the signal can be represented by $\mathrm{N} / \mathrm{m}$ components and the coefficients are $\left[\begin{array}{llll}c_{0} & c_{1} & \ldots & c_{N / m}\end{array}\right]$. However, when there is only noise in the signal, the total $N$ coefficients are nonzero. Since the transformation is orthogonal, the energy after the transformation is the same as that before the calculation, so we can obtain $\left(S \mid H_{0}\right) \sim N\left(0, \sigma^{2} / m\right)$ and

$$
\left(S \mid H_{1}\right) \sim N\left(E, \sigma^{2} / m\right),
$$

where $E$ is the power of the PU signal $x(t)$ in the received signal. Then, the probability density function (PDF) of $S$ can be expressed as equation (7),

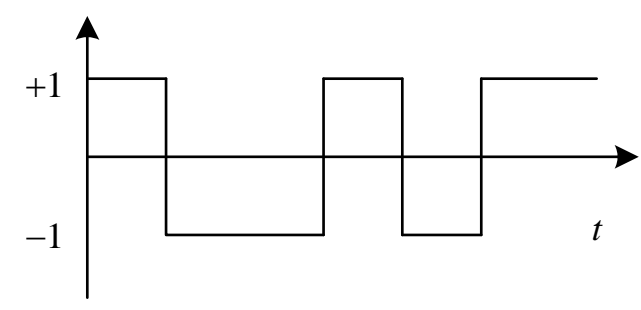

Figure 1. Example of BPSK signal.

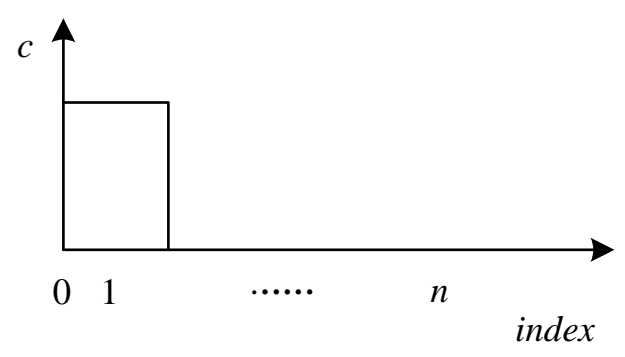

Figure 2. Obtained $\left\{c_{k}\right\}$ after Walsh transformation. 


$$
\begin{aligned}
& p\left(S \mid H_{0}\right)=\sqrt{\frac{m}{2 \pi \sigma^{2}}} \exp \left(-\frac{m S^{2}}{2 \sigma^{2}}\right) \\
& p\left(S \mid H_{1}\right)=\sqrt{\frac{m}{2 \pi \sigma^{2}}} \exp \left(-\frac{m(S-E)^{2}}{2 \sigma^{2}}\right)
\end{aligned}
$$

Then the false alarm probability can be derived,

$$
\begin{aligned}
P\left(H_{1} \mid H_{0}\right) & =\int_{t}^{\infty} p\left(S \mid H_{0}\right) d S \\
& =\int_{\frac{\sqrt{m}}{\sigma} t}^{\infty} t \sqrt{\frac{1}{2 \pi}} \exp \left(-\frac{l^{2}}{2}\right) d l \\
& =Q\left[\frac{\sqrt{m}}{\sigma} t\right],
\end{aligned}
$$

where

$$
Q\left[u_{0}\right]=\int_{u_{0}}^{\infty} \sqrt{\frac{1}{2 \pi}} \exp \left(-\frac{u^{2}}{2}\right) d u .
$$

Similarly, the detection probability can also be obtained,

$$
\begin{aligned}
P\left(H_{1} \mid H_{1}\right) & =\int_{t}^{\infty} p\left(S \mid H_{1}\right) d S \\
& =Q\left[\frac{\sqrt{m}}{\sigma}(t-E)\right] .
\end{aligned}
$$

From the above equations, the probability of false alarm can be calculated for a given decision threshold from equation (8), which can also be used to obtain the threshold if the probability of false alarm is constrained.

\section{Simulation Results}

In this section, the performance of the proposed detector in the present paper is compared with the spectral feature detector in [4] through simulation.

The baseband signal used in the simulation is BPSK signal with rectangle envelope. The symbol rate is $2 \mathrm{MB}$ and the sample frequency is $32 \mathrm{MHz}$. A couple of numerical experiments are carried out and a block of received data with 10240 samples is generated to calculate a decision result for every experiment.

Figure 3 shows the variation of probability of detection with probability of false alarm for BPSK when SNR is $-18 \mathrm{~dB}$. The probability of detection decreases as the probability of false alarm falls. When the false alarm probability is given, the proposed method has higher detection

Figure 4 illustrates the effect of SNR on the probability of detection for BPSK when the probability of false alarm is 0.01 . It is showed that the probability of detection decreases as SNR falls when the probability of false alarm is fixed. When SNR is given, the proposed method has higher detection probability.

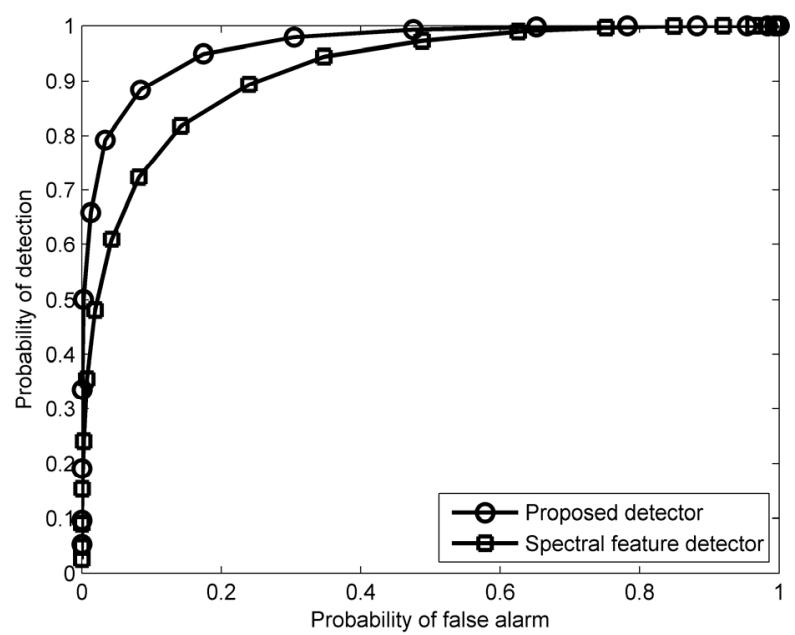

Figure 3. Probability of detection versus probability of false alarm for BPSK when SNR is -18 dB.

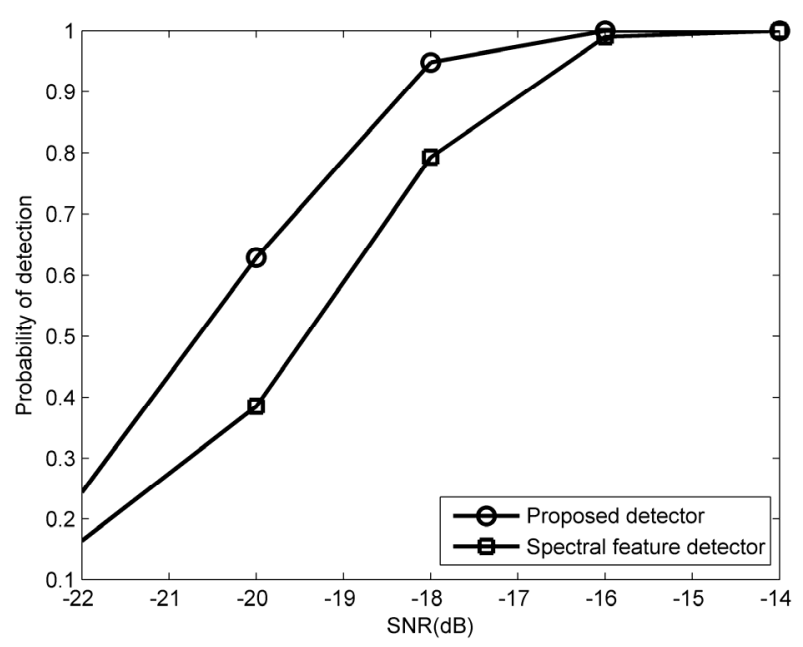

Figure 4. Probability of detection versus SNR for BPSK when the probability of false alarm is $\mathbf{0 . 0 1}$.

\section{Conclusions}

In this paper, a feature detector based on Walsh transform is proposed, which exploits the feature of the signal after Walsh transformation to decide the presence of the useful signal. It has been proved that the proposed detector performs better than the spectral feature detector at low SNR by the simulation results. In addition, the proposed method is low in complexity, which makes it typically suitable for real time spectrum sensing in cognitive radio system.

\section{REFERENCES}

[1] T. Cui, J. Tang and F. Gao, "Blind Spectrum Sensing in Cognitive Raio," in Proc. IEEE Wireless Communications and Networking Conference (WCNC). 2010.

[2] S. Haykin, “Cognitive Radio: Brain-empowered Wireless 
communications," IEEE J. Sel. Areas Commun, Vol. 23, 2005, pp. 201-220. doi:10.1109/JSAC.2004.839380

[3] F. F. Digham, M. S. Alouini and M. K. Simon, "On the Energy Detection of Unknown Signals over Fading Channels," IEEE International Conference on Communications (ICC'03), pp. 3575-3579, May 2003.

[4] Z. Quan, W. Zhang, S. Shellhammer and A. H. Sayed, "Optimal Spectral Feature Detection for Spectrum Sensing at very Low SNR," IEEE Transactions Communications, Vol. 59, 2001, pp. 201-212.

doi:10.1109/TCOMM.2010.112310.090306
[5] W. A. Gardner and C. M. Spooner, "Signal Interception: Performance Advantages of Cyclic Feature Detectors," IEEE Transactions Communications, Vol. 40, 1992, pp. 149-159. doi:10.1109/26.126716

[6] K. G. Beauchamp, application of Walsh and Related Functions. New York: Academic, 1984.

[7] R. F. Mills and G. E. Prescon, "A Comparison of Various Radiometer Detection Models," IEEE Transactions on Aerospace and Electronic Systems, Vol. 32, No. 1, 1996, pp. 467-473. doi:10.1109/7.481289 